\title{
Stem Cells and Regeneration: Special Review Issue
}

\author{
Renee A. Reijo Pera and \\ Department of Obstetrics and Gynecology, Center for Human Embryonic Stem Cell, Research \\ and Education, Institute for Stem Cell Biology and Regenerative Medicine, Stanford University \\ School of Medicine, Stanford, USA

\section{Joseph G. Gleeson} \\ Investigator, Howard Hughes Medical Institute, Departments of Neurosciences and Pediatrics, \\ University of California, San Diego, USA
}

\begin{abstract}
When the editorial board of Human Molecular Genetics was queried to consider potential topic areas for the annual 'special review', one topic that was enthusiastically endorsed was 'stem cells and regenerative medicine'. The fusion of these two topics reflected the hope and potential that advances in regenerative medicine may be the primary outcome of much of the current basic and translational research in the field of stem cell researches.
\end{abstract}

The goal in soliciting input for this special issue was to capture the current thinking, and identify priorities, in stem cell biology as it relates specifically to human molecular genetics. The order of the articles in this issue approximates the timeline of embryonic development toward adulthood and then regeneration. Many of the important germline derivatives are covered in this review, to include pluripotent embryonic stem cells (ESCs) and their genetic regulation, animal ESC models and potential applications. Organ- or tissue-specific applications encompass dermal and endocrine derivatives, applications toward neural and skeletal diseases and potential therapies in regeneration. In order to capture a glimpse of the current view of stem cell and regenerative medicine, we asked several leaders in stem cell biology to comment on what they considered the most important issues currently facing the fields of stem cell and regeneration.

The historical perspective and broad agenda are described in a particularly insightful review by Christopher Scott, Director of the Program on Stem Cells in Society, and Renee Reijo Pera, Director of the Center for Human Embryonic Stem Cell Research and Education, both at Stanford. This story begins with a discussion of the controversy surrounding the historical decision by the US Executive Branch to ban funding for research on human embryos and how this has shaped research on human ESCs (hESCs) researches. The article weaves through the important controversies that have altered the current landscape of hESC research. The issue continues with a review by Amander Clark and Gayane Ambartsumyan from the Broad Center of Regenerative Medicine and Stem Cell Research at UCLA that lays the groundwork for a fascinating approach to model human aneuploidies (such as germline trisomies and monosomies) via the use of hESCs. This could provide a novel approach for 
probing human chromosomal nondisjunction, since it could elucidate unique cell cycle and checkpoint requirements in the early human embryo. The journey continues with the article authored by Niels Geijsen from the Center for Regenerative Medicine and Technology at Massachusetts General Hospital, Harvard University, and Leanne Jones at the Laboratory of Genetics, Salk Institute. These researchers merged their ideas and reviewed the current understanding of male germline cell differentiation, their unique environmental and genetic profiles, and their relevance in the study to pluripotent stem cell and regenerative biology. George Daley, current President of the International Society for Stem Cell Research (ISSCR) and a member of the Harvard Stem Cell Institute/Children's Hospital, Boston, then provides an assessment of pluripotency, and the transcriptional and regulatory factors responsible for its maintenance. This theme is continued with the review authored by Geof Rosenfeld and Victoria Lunyak from the Howard Hughes Medical Institute at UCSD as they describe the 'epigenetic instruction manual' for stem cell biology, by reviewing the remarkable progress in understanding chromatin properties and histone modifications as well as other regulatory mechanisms under the influence of key factors such as OCT4 and NANOG. Their own work in identifying functionally important co-repressors in maintenance of stem cell fate has opened up new avenues for understanding this regulation of cellular identity.

As we progress through this special issue, James Byrne, from the Center for Human Embryonic Stem Cell Research and Education in the Institute of Stem Cell Biology and Regenerative Medicine at Stanford, reviews the current methodologies for reprogramming primate somatic cells into pluirpotent stem cells. Future cell-based therapies will surely benefit from isogenic transplantation (i.e. cells from one patient, reprogrammed and differentiated for transplantation to that patient). This is a critical issue that has been the subject of intense research in the last few years. If cell-based therapy is to reach its full potential, understanding of the cellular capacity for reprogramming, and continued comparison between methods, is critical. This review naturally precedes that of Marlon Schneider from the Institute of Molecular Animal Breeding and Biotechnology, University of Munich, which considers the importance of large animal models for understanding disease and testing therapies. The canine is proposed as a model for testing cell-based therapies as the review discusses disease models and progress in establishing canine pluriopotent stem cells. The subject of pluripotent stem cells, especially challenges and early solutions for the manufacture of clinical-grade hESCs, is further expounded upon by Outi Hovatta et al. from the Department of Clinical Science, Intervention and Technology at the Karolinska Institute. Their valuable perspective comes from years of working in close contact with a clinical human in vitro fertilization facility, and in contemplating methods to move cells into clinical applications.

Specialized somatic derivatives are the subject of subsequent reviews beginning with that provided by Ben $\mathrm{Yu}$, a dermatologist and molecular geneticist from UCSD, who discusses the potential role for stem cell therapy in treating disorders of the skin, and the current knowledge of inducing dermal subtype differentiation. Julie Gaulden and Jeremy Reiter from the Cardiovascular Research Institute and Human Embryonic Stem Cell Research Center at UCSF then discuss the influences of extracellular signals such as bone 
morphogenetic proteins (BMPs), fibroblast growth factors (FGFs) and wingless and int family (Wnts) in regulating stem cell fates, particularly in regards to the developing neuronal lineage. Fred Gage et al. at the Laboratory of Genetics, Salk Institute discuss the multiple layers of control in the pathway from ESC to neuron, including chromatin modification, methylation, microRNA and posttranscriptional and posttranslational regulation of neuronal fate. With so many unique types of cells in the nervous system, the review provides a guide for future challenges of cell-based therapies in the neurosciences. Steve Goldman et al. from the Division of Cell and Gene Therapy and Center for Translational Neuro-medicine at the University of Rochester discuss how they have overcome the challenges in developing treatment strategies for myelin central nervous system disorders using cell-based therapy. They further discuss disorders such congenital central hypomyelination/leukodystrophies and their success with transplantation of human neural cells into the shiverer mouse mutant. Transplantation is further discussed by Zhiguo Chen and Theo Palmer, Institute for Stem Cell Biology and Regenerative Medicine and Neurosciences Institute at Stanford, as they describe practical considerations of stem cell grafts into the central nervous system, and raise the limitations and challenges faced while overcoming immunological responses. Finally, we close with the review by Michael Longaker from the Institute of Stem Cell Biology and Regenerative Medicine at Stanford. Dr Longaker is a leader in osteogenic regeneration, and describes the frontiers of this exciting field, including evolving models to test the effects of adipose-derived stromal cellbased therapies in skeletal injuries and disorders.

We would be hard-pressed to find a field moving forward more rapidly, which has benefited from the focused public eye (and public funding), and which has elicited great expectations for clinical efficacy and utility. Stem cell biology and regenerative medicine is an area of biomedical sciences where some of the most exciting discoveries may be disseminated prior to full peer review and publication, and where information about new methodologies or important advances may spread like wildfire prior to validation. We hope that this timely compendium of reviews by scientific leaders may positively impact this field, through the introduction of new ideas and recognition of the common challenges that lay ahead in a well-balanced manner. Clearly, the field has a responsibility to communicate openly and fairly about advances, as well as failures, so that the public might reap the maximal benefits of this exciting emerging field. 